\title{
Two different approaches to an analytical chemistry laboratory practical: wider and shallower or narrower and deeper?
}

\author{
Nataša Gros ${ }^{1}$ \\ Published online: 16 July 2019 \\ (C) Springer-Verlag GmbH Germany, part of Springer Nature 2019
}

\section{Introduction}

The laboratory practical has an important role in shaping prospective analytical chemists. Students become familiar with the practical aspects of the discipline and can better understand what they listened to during the lectures. However, of at least equal importance are their attitudes to laboratory work and the practical skills they develop.

Learning how to perform laboratory operations correctly, being capable of working in an organised and well-considered way, following written procedures, and documenting processes adequately are aspects of good laboratory practices (GLPs), also abbreviated GLP by some authors [1], to distinguish them easily from good laboratory practice, GLP, which denotes a formal regulative document of the US Food and Drug Administration [2]. GLP in contrast to GLPs is a document including a set of official requirements that pharmaceutical studies should adhere to, to make their findings acceptable to official bodies. A clear link between the raw data and the findings should be established through the documentation.

Nevertheless, as William Horwitz pointed out, both are connected, since good practices in analytical chemistry are more a matter of common sense than an artificial bureaucratic burden, as some people were claiming when GLP was introduced [3]. Good practices reduce error rates, contribute to the quality of the analytical results and make analytical chemists more reliable and more efficient when conducting research work or routine analyses. After all, every quality-regulation scheme requires skilled and resourceful personnel.

Nataša Gros

natasa.gros@ fkkt.uni-lj.si

1 Faculty of Chemistry and Chemical Technology, University of Ljubljana, Večna pot 113, Ljubljana, Slovenia
Good practices do not develop in students automatically, just by them being involved in practical work. The students need to be taught about these practices and trained in them, so that they become second nature. There are a variety of approaches to achieving this. Here, we describe and compare two variants of analytical chemistry laboratory practicals and discuss their influence on the students' self-efficacy beliefs (SEBs) and awareness of GLPs.

Our inspiration came from an observation that a narrowerand-deeper approach might be more profitable for the students than a wider-and-shallower one. We noticed this in 2017, when we evaluated how well prepared for different aspects of analytical chemistry the students of different programmes consider themselves to be at the end of their education in analytical chemistry. The third-year students of the first Bologna cycle in Chemistry and a vocational programme in Chemical Technology, and the first-year students of the programmes Laboratory Biomedicine, first Bologna cycle, and Pharmacy, unified first and second Bologna cycles, were involved.

The major difference between the groups of younger students was observed in the SEBs of being able to manipulate analytical instruments correctly, in which the students of Laboratory Biomedicine exhibited the highest confidence of all the groups. We found this surprising, since in their study programme, only $14 \mathrm{~h}$ of the laboratory practicals, out of 30 in total, was devoted to instrumental methods, the lowest share of all the groups. It seemed that the way they were treated had contributed. We decided to re-examine the SEBs of the students of Laboratory Biomedicine and Pharmacy in 2018 to evaluate how significant the differences between the two approaches are. We additionally used a pre- and a post-test to measure the impact of the laboratory practical on improving the students' awareness of GLPs after completing a practical in analytical chemistry. 


\section{Methods}

\section{Participants and procedures}

During the second semester, students of the first Bologna cycle programme of Laboratory Biomedicine and a uniform master's degree study programme of Pharmacy have 6 and 8 ECTS (European Credit Transfer System), respectively, allocated to Analytical Chemistry. The education provided to the students by analytical chemists finishes at the end of the first year. Analytical chemistry topics are included in other subjects, which brings the programmes closer to 15 ECTS, as suggested by Eurocurriculum II, but they are not taught by analytical chemists [4].

Both programmes have analytical chemistry laboratory practicals integrated into a module. The share of the contact hours between lectures, seminars and laboratory practicals is different and specified in Table 1. The students of the two programmes listen to the lectures separately, although the lecturer is the same.

Approximately 150 students of Pharmacy and usually between 40 and 50 students of Laboratory Biomedicine enter the programme. Both programmes are oversubscribed and consequently only available to students with excellent results at the pre-university level.

A typical number of students in a group for a laboratory practical is 15. Due to the large number of groups, the students of Pharmacy have the first 3 days of a week mainly dedicated to practicals for different subjects, and the remaining two to lectures. The practicals in analytical chemistry start for them simultaneously with the lectures, and last for 10 weeks.

In the timetable for Laboratory Biomedicine, only $2 \mathrm{~h}$ per week $(2 \times 45 \mathrm{~min})$ is dedicated to analytical chemistry practicals. The time is far too short to perform analytical work. Therefore, the contact hours had to be reorganised so that during the first 6 weeks, there are $6 \mathrm{~h}$ of lectures, during the following 3 weeks, $3 \mathrm{~h}$ of lectures and $4 \mathrm{~h}$ of practicals, and during the final 4 weeks, practicals only. Rescheduling made it possible to introduce the theoretical background during the lectures, before it is required for the practicals.

\section{Instruments and data evaluation}

We developed a paper-form questionnaire in which students graded their SEBs concerning different analytical chemistryrelated competencies and practical skills on a scale from 0 to $100 \%$ competent. The opening phrase was: "How well prepared do you consider yourself at the end of a course for ...." The questionnaires were presented to the students during supervised sessions close to the end of the second semester. The students were asked to respond individually.

We created a pre- and a post-test to measure the potential improvement in the students' awareness of GLPs after completing a practical in analytical chemistry. The pre-test comprised 19 closed-ended four-choice questions, 10 of which were used in the post-test, one of which had to be adapted to prevent the effect of memorising. The questions were elected for a post-test, first, if they were considered predominantly practical influenced and, next, if a low awareness was observed for at least one group of students, or the topic was considered very important. The Kahoot online voting system was used during the lectures. Neither of the tests was announced in advance and the students did not prepare for any of them. In order to ensure that the practical was guided as usual, and that the staff did not influence the outcomes through their own intentions, they were not made aware of the testing taking place during the lectures. The percentage of correct answers was calculated from the number of participants in the online quiz and not from the number of responses to a particular question.

The data were evaluated with MS Excel and Statgraphics Centurion XVI.

\section{Results and discussion}

\section{Comparison of the two versions of the analytical chemistry laboratory practical}

The first 5 weeks of the laboratory practical for students of Pharmacy is dedicated to titrimetric methods. During the subsequent 5 weeks, they perform instrumental analyses. The programme is summarised in Table 2. The focus is on determinations and report writing. Due to the fact that the lectures and the practicals start simultaneously, the students generally perform the laboratory activities before a topic is introduced during the lectures. Consequently, the laboratory instructions need to comprise the essential background, while the directions for practical work are brief, and general GLPs are not discussed. For practical aspects and the use of instrumentation, the students depend on staff support, oral instructions, demonstrations or interventions.

By rescheduling the timetable, it was possible to ensure for the Laboratory Biomedicine students that laboratory activities

Table 1 Characteristics of the analytical chemistry module in the study programmes

\begin{tabular}{|c|c|c|c|c|c|c|}
\hline Programme & Cycle & Duration & Analytical chemistry & Lectures & Seminars & Laboratory practicals \\
\hline Laboratory Biomedicine & $1 \mathrm{st}$ & 3 years & 6 ECTS* & $45 \mathrm{~h}$ & - & $30 \mathrm{~h}$ \\
\hline Pharmacy & $1 \mathrm{st}+2 \mathrm{nd}$ & 5 years & 8 ECTS* & $45 \mathrm{~h}$ & $15 \mathrm{~h}$ & $45 \mathrm{~h}$ \\
\hline
\end{tabular}

* Delivered by analytical chemists 
Table 2 Activities performed by students of Pharmacy during the instrumental-analysis sessions

\begin{tabular}{ll}
\hline $\begin{array}{l}\text { Class } \\
\text { duration }\end{array}$ & Activity \\
\hline $4 \mathrm{~h}$ & $\begin{array}{c}\text { Fluoride determination with ion-selective electrode (ionic } \\
\text { strength not adjusted) }\end{array}$ \\
$4 \mathrm{~h}$ & $\begin{array}{l}\text { Iron determination with molecular absorption spectrometry } \\
\text { Quinine determination with molecular fluorescence } \\
\text { spectrometry and the method of standard additions }\end{array}$ \\
$4 \mathrm{~h}$ & $\begin{array}{c}\text { Sodium determination in a physiological solution with } \\
\text { flame-emission spectrometry } \\
\text { Copper and manganese determination in a mineral-vitamin } \\
\text { tablet with atomic absorption spectrometry }\end{array}$ \\
& $\begin{array}{c}\text { Caffeine determination in soft drinks with } \\
\text { high-performance liquid chromatography }\end{array}$ \\
\hline
\end{tabular}

take place after the presentation of the theory. This made things easier; however, the challenge of how to make an impact with a 30 school hours (22.5 full hours) of practicals remained.

We decided to dedicate the first session to gravimetric analyses, the following three to titrimetric methods, the next one to a total analytical process, and the remaining two to instrumental methods. Due to the very limited times for instrumental analyses in the programme, we opted for a more in-depth approach and limited ourselves to just molecular absorption spectrometry and potentiometry. The activities of the last three sessions are more precisely described in Table 3 . In comparison with Table 2, it is obvious that the workload is much higher for the students of Laboratory Biomedicine than for the students of Pharmacy. In order to achieve higher efficiency, appropriate support was needed.

We decided to write a comprehensive textbook for the laboratory practicals that would introduce the students to GLPs and would be detailed and instructive enough to make the students as independent as possible when performing the laboratory activities and handling the instrumentation [5]. The students are required to read the content carefully, and well in advance, to become familiar with the approaches that they are going to use. There is not enough time to start reading the materials during the sessions. The theory is not repeated, the students are expected to revise it through an inquiry-based approach as a preparation for the laboratory work. The textbook is also a workbook. The symbol of a watch indicates to the students what they need to do in advance in the form of preparation; a notebook symbol indicates where the results ought to be recorded, treated or graphically presented; and a flag symbol points out where interpretations, explanations or conclusions should be presented. The students are encouraged to fill in as much as possible during the laboratory sessions. They are not required to write an extra report afterwards.

The students of Laboratory Biomedicine receive information about the accuracy of their results in the form of
Table 3 Activities performed by students of the Laboratory Biomedicine during the instrumental-analysis sessions

$\begin{array}{ll}\text { Class } & \text { Activity } \\ \text { duration } & \end{array}$

$4 \mathrm{~h}$

Evaluation of a natural water source - total analytical process from a study of the context, statement of a hypothesis, field sampling, laboratory determinations, to interpretation of results within the context of all the results obtained by all the students within the same laboratory session (calcium, chloride, hydrogen phosphate, nitrite determination with a tri-colour,

light-emitting-diode-based spectrometer, hydrogen carbonate and total water-hardness determination with a simplified and miniaturised titration set-up, $\mathrm{pH}$ determination with a field instrument)

$5 \mathrm{~h}$

Molecular absorption spectrometry

- Absorption spectrum (observing light colour and wavelength, colour of solution and light absorption, manual and automatic recording of a spectrum)

- Instrument qualification (wavelength and absorbance accuracy, linearity and stray-light check)

- Spectrometric determination of a single analyte in a sample

- Spectrometric determination of two analytes in a sample by two wavelength-absorbance measurements

$5 \mathrm{~h}$

Potentiometry

- Fluoride-ion determination in highly mineralised water (with and without ionic strength adjustment)

- Two-point calibration of a $\mathrm{pH}$ meter

- Determining a sample $\mathrm{pH}$ accurately

- Checking the electrode measurement range

- Preparation of a buffer and evaluation of its $\mathrm{pH}$

- Determining buffer's composition with a $\mathrm{pH}$ measurement and potentiometric titration

control charts comprising the results of all the students in a group. They can compare their own achievements with those of the others and are at the same time becoming familiar with the charts, which are an essential part of quality control. Each result within a $\pm 5 \%$ tolerance limit increases the mark for the student concerned with respect to a particular degree.

The practical continues with the activity "Evaluation of a natural water source", as a student's individual mini-project (Table 3), which raises the interpretation of the results to a higher level. Each student chooses a water source that he or she knows well and would like to examine, gathers information about the geology, natural environment and possible human-related influences, states the hypotheses and then performs the field sampling. The students bring the samples to the laboratory and they exchange working places so that each student determines seven parameters in his/her own water sample. They collect and obtain the results for all the samples. They write an essay and discuss the characteristics of their water source with respect to the background they studied initially, and hopefully also within the wider context of the characteristics of the other water sources. They are encouraged to 
Table 4 Self-efficacy beliefsthe means of the groups and differences confirmed with $95.0 \%$ Fisher's least-significantdifference test (95.0\% LSD)

\begin{tabular}{lllll}
\hline Ability to & Programme & Count & Mean & Homogeneous groups \\
\hline -Perform laboratory operations correctly & FARM 2017 & 60 & 66.0 & $\times$ \\
& FARM 2018 & 80 & 69.6 & $\times$ \\
& LBM 2017 & 31 & 80.5 & $\times$ \\
& LBM 2018 & 37 & 77.8 & $\times$ \\
-Communicate with analytical chemists & FARM 2017 & 60 & 60.6 & $\times$ \\
& FARM 2018 & 80 & 64.2 & $\times$ \\
& LBM 2017 & 31 & 74.7 & $\times$ \\
& LBM 2018 & 37 & 75.3 & $\times$ \\
& FARM 2017 & 60 & 59.0 & $\times$ \\
-Work with instrumentation & FARM 2018 & 80 & 61.6 & $\times$ \\
& LBM 2017 & 31 & 66.0 & $\times \times$ \\
& LBM 2018 & 37 & 69.9 & $\times$ \\
& FARM 2017 & 60 & 60.9 & $\times$ \\
& FARM 2018 & 80 & 65.5 & $\times \times$ \\
& LBM 2017 & 31 & 72.1 & $\times$ \\
& LBM 2018 & 37 & 70.4 & $\times$
\end{tabular}

relate and compare the different parameters. They evaluate their hypotheses and write conclusions. The quality of their essay contributes to their mark.

During the instrumental sessions, the students become acquainted with different aspects of molecular absorption spectrometry and potentiometry (Table 3 ). They are divided into smaller groups and rotate between the different working places. They deal with instruments that have different designs and sophistication independently, by following written instructions in the textbook/workbook, and treat, present and interpret the data as required.

\section{Students' responses—self-efficacy beliefs}

Students graded their SEBs as a percentage. The group means regarding the four different abilities are summarised in Table 4. The abbreviations FARM or LBM stand for the groups of students from Pharmacy or Laboratory Biomedicine, respectively. A year indicates a generation. For all the abilities, the means of the LBM were higher than those of the FARM.

A $95.0 \%$ Fisher's least-significant-difference procedure (95.0\% LSD) was used to recognise the significant differences among the means of the groups of students. The test confirmed that for each of the four statements FARM 2017 and 2018 represent homogenous groups, and the same is true for LBM 2017 and 2018. However, students of LBM and FARM, no matter which generation, present two distinct groups concerning the SEBs of being able to perform laboratory operations correctly or communicate with analytical chemists. For the SEBs of being able to work with instrumentation, the mean of LBM 2018 was significantly higher than the means of both generations of FARM. Concerning the ability to treat and interpret data independently, both generations of LBM exhibit significantly higher confidence than FARM
Fig. 1 Results of the pre- and the post-test concerning students' awareness of GLPs related to instrumental methods

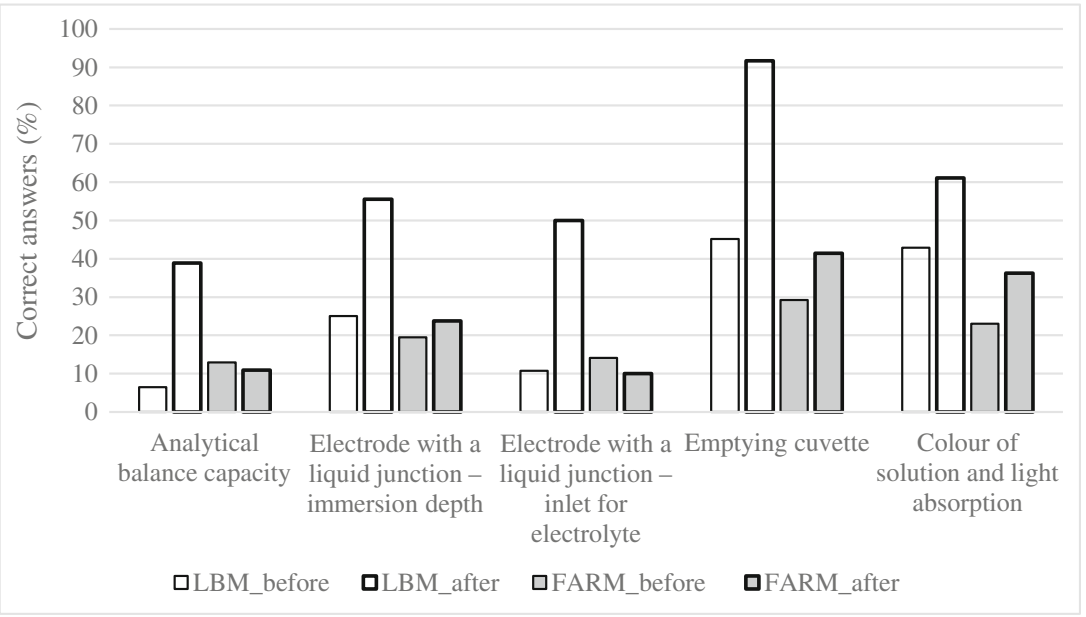


Fig. 2 Results of the pre- and the post-test evaluation of the students' awareness of GLPs concerning fundamental laboratory skills

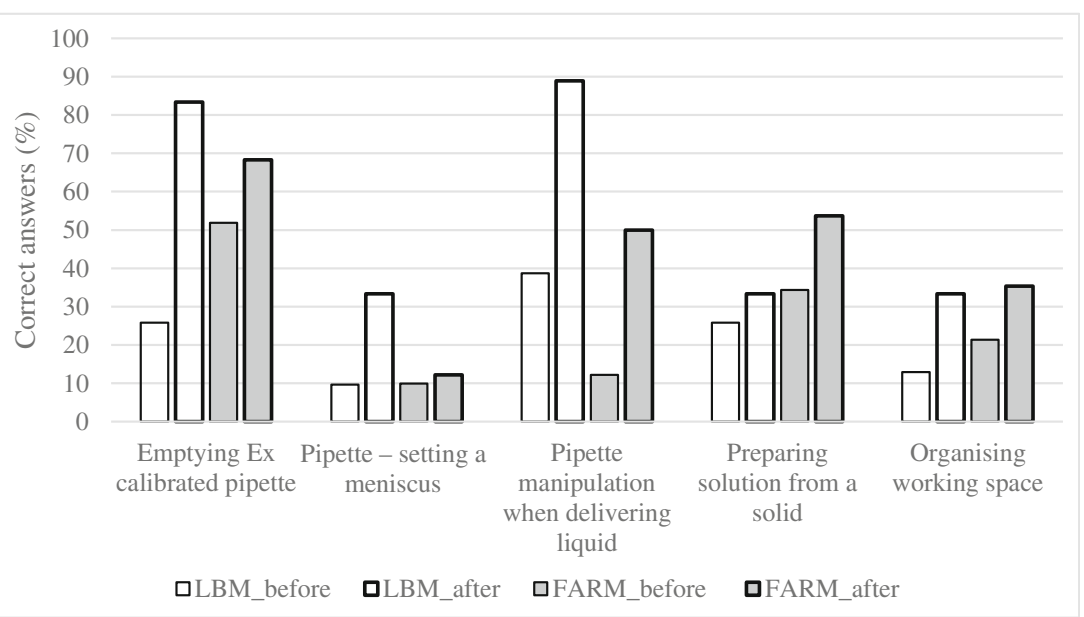

2017. We can conclude that the way students were treated contributed to their SEBs. A deeper-and-narrower approach proved more beneficial to the students' SEBs than the widerand-shallower one. Several authors consider that higher SEBs contribute to students' performance and achievements [6-9]. In the continuation, we examined to what extent the laboratory practical influenced the students' awareness of GLPs.

\section{Students' awareness of good laboratory practices}

In Figs. 1 and 2, we compare the percentage of correct answers for the pre- and the post-test for the two programmes. The empty bars relate to LBM, and the grey bars to FARM. A bold bar outline indicates the post-test results for both programmes. It should be mentioned that the students of Pharmacy previously took part in a practical in general chemistry. In contrast, for the students of Laboratory Biomedicine, this was the first chemical-laboratory experience.

The aspects of the analytical balance's weighing capacity, the preparation of a solution from a solid, the use of volumetric equipment, the handling of an electrode with a liquid junction, the emptying of a spectrometric cuvette correctly, the choice of a light source in relation to the colour of a solution, and organising a working space were covered. The general observation based on Figs. 1 and 2 is that the students of Laboratory Biomedicine made more progress than the students of Pharmacy. Their awareness of GLPs usually improved to a greater extent, and they reached a higher percentage of correct answers in the post-test than the students of Pharmacy.

For LBM, the highest 80 to $90 \%$ awareness of GLPs was observed for cuvette manipulation, correct use of the excalibrated pipette, and pipette manipulation when delivering a liquid. Similarly, the latter two aspects were also among the best post-test achievements for FARM, with 68 and $50 \%$, respectively.
In contrast to what was already discussed concerning volumetric operations, a misconception was recognised in relation to the meniscus setting. The improvement from the pre- to the post-test was only from 10 to $33 \%$ of the correct answers for LBM, and no real improvement at all for FARM, with 10 and $12 \%$ of the correct answers, respectively. The students mainly did not recognise that while setting a meniscus, delivering the solution back into the same vessel from which it was initially drawn is not an aspect of GLPs.

A different pattern than generally encountered was observed for the preparation of a solution from a solid in which FARM with $54 \%$ of correct answers outperformed the students of LBM, who reached $33 \%$. Another exception is organisation of the working space, in which students of both programmes in the post-test achieved a similar percentage of correct answers, close to $35 \%$. In this case, it turned out that the improvement was more likely caused by the lectures than the practical.

The pre- to post-test-performance comparison of the programmes proved that the way the students were treated during the practical contributed to their awareness of GLPs. At the same time, the higher SEBs in the students of Laboratory Biomedicine did not turn out to be without foundation, as they are related to a better awareness of GLPs.

\section{Conclusions}

This study confirmed that GLPs do not develop in students automatically, just by being involved in practical work. Students need to be taught about them and trained in them to make them second nature, and that a narrower and more indepth approach proved to be beneficial. The laboratory work instructions for Laboratory Biomedicine [5] were comprehensive and much more extensive than those of a comparable group from Pharmacy, and allowed the students to work independently, more intensively and with as little intervention 
from the staff as possible. GLPs received proper attention. A mini-project covering the total analytical process, data interpretation within a wider context, control charts stimulating the quality of the analytical work, and experiences with instruments of different designs and sophistication were part of the approach. As a result, higher SEBs associated with better awareness of GLPs were observed in students of Laboratory Biomedicine than in students of Pharmacy.

\section{References}

1. Prichard E, Barwick V. Quality assurance in analytical chemistry. Analytical techniques in the sciences: John Wiley \& Sons, Ltd; 2007.

2. McDowall RD. What does good laboratory practice (GLP) mean. Lc Gc Europe. 2008;21(6):339-+.

3. Horwitz W. Good laboratory practices in analytical-chemistry. Anal Chem. 1978;50(6):A521-A4. https://doi.org/10.1021/ac50027a001.

4. Salzer R. Eurocurriculum II for analytical chemistry approved by the division of analytical chemistry of FECS. Anal Bioanal Chem. 2004;378(1):28-32. https://doi.org/10.1007/s00216-003-2358-3.

5. Gros N. Analizna kemija: univerzitetni študijski program Laboratorijska biomedicina. Učbenik za laboratorijski del predmeta. Ljubljana: Univerza v Ljubljani, Fakulteta za kemijo in kemijsko tehnologijo; 2015.

6. Ouweneel E, Schaufeli WB, Le Blanc PM. Believe, and you will achieve: changes over time in self-efficacy, engagement, and performance. Appl Psychol Health Well Being. 2013;5(2):225-47. https:// doi.org/10.1111/aphw.12008.

7. Domenech-Betoret F, Abellan-Rosello L, Gomez-Artiga A. Self-efficacy, satisfaction, and academic achievement: the mediator role of students' expectancy-value beliefs. Front Psychol. 2017;8. https:// doi.org/10.3389/fpsyg.2017.01193.
8. Komarraju M, Nadler D. Self-efficacy and academic achievement: why do implicit beliefs, goals, and effort regulation matter? Learn Individ Differ. 2013;25:67-72. https://doi.org/10.1016/j.lindif.2013. 01.005 .

9. Kapucu S, Bahcivan E. High school students' scientific epistemological beliefs, self-efficacy in learning physics and attitudes toward physics: a structural equation model. Res Sci Technol Educ. 2015;33(2):252-67. https://doi.org/10.1080/02635143.2015. 1039976.

Publisher's note Springer Nature remains neutral with regard to jurisdictional claims in published maps and institutional affiliations.

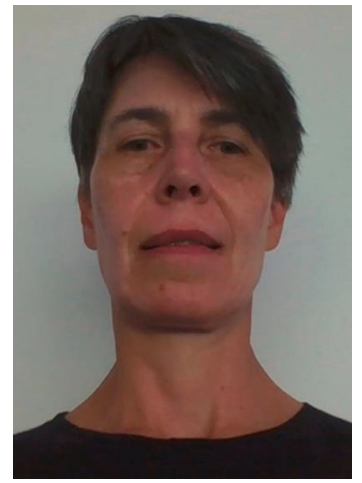

Nataša Gros is the Associate Professor of Analytical Chemistry at the Faculty of Chemistry and Chemical Technology of the University of Ljubljana. Her scientific interests include development of smallscale low-cost analytical instruments, measurement uncertainty and quality control, ion chromatography and water analyses. She coordinated two European Leonardo da Vinci projects entitled Hands-on Approach to Analytical Chemistry for Vocational Schools (AnalChemVoc, AnalChemVoc II); the second was declared one of the best projects of a call of the year. At the Division of Analytical Chemistry of the European Chemical Society, EuChemS, she represents the Slovenian Chemical Society. 